\title{
Echinococcus granulosus: Cure for Cancer Revisited
}

\author{
Shiwanthi L. Ranasinghe* and Donald P. McManus \\ Molecular Parasitology Laboratory, Department of Immunology, QIMR Berghofer Medical Research Institute, Brisbane, QLD, \\ Australia
}

Whereas a number of parasites are well recognized risk factors for a number of different cancers in mammalian hosts, there is limited information on the ability of parasitic organisms to induce anticancer effects. There are conflicting reports that echinococcosis, caused by the canine tapeworm Echinococcus granulosus, can decrease or increase cancer risk. This review considers both indirect anticancer effects as the result of adaptive immunity generated against certain echinococcal antigens and the direct effect of molecules released by $E$. granulosus whose activity directly inhibits cancer cell migration and growth. In conclusion, E. granulosus probably secretes molecules that can be developed as anticancer therapeutics in future.

Edited by: Monica Catarina Botelho, Instituto Nacional de Saúde

Doutor Ricardo Jorge (INSA), Portugal

Reviewed by: Ruben Fernandes, Escola Superior de Saúde do Porto, Politécnico do Porto, Portugal Daniel Ferreira Feijó, Instituto Gonçalo Moniz (IGM), Brazil

${ }^{*}$ Correspondence: Shiwanthi L. Ranasinghe shiwanthi.ranasinghe@ qimrberghofer.edu.au

Specialty section: This article was submitted to Infectious Diseases - Surveillance,

Prevention and Treatment, a section of the journal Frontiers in Medicine

Received: 30 October 2017 Accepted: 19 February 2018

Published: 12 March 2018

Citation:

Ranasinghe SL and McManus DP (2018) Echinococcus granulosus: Cure for Cancer Revisited. Front. Med. 5:60. doi: 10.3389/fmed.2018.00060
Keywords: Echinococcus granulosus, cancer therapy, Kunitz type protease inhibitor, antibody-mediated response, Echinococcus antigens

\section{INTRODUCTION}

There is increasing evidence that some infectious agents induce antitumor activity against different types of cancers (1). Experiments in vitro have shown that certain parasites including the protozoans Trypanosoma cruzi (2), Toxoplasma gondii (3), and Acanthamoeba castellanii (4) and the helminths Echinococcus granulosus (5) and Strongyloides stercoralis (6) exhibit anticancer activities. However, there are conflicting reports in the literature that the canine tapeworm (Phylum Cestoda) E. granulosus, the cause of human cystic hydatid disease (echinococcosis) in many parts of the world is able to reduce cancer growth (7-9). A significantly lower prevalence of cancer was reported in patients with hydatid disease in a large retrospective study in Turkey (7). In direct contrast, a pilot retrospective study carried out in Cyprus indicated that echinococcosis may increase cancer risk in patients (10). In the main, there is more evidence to support the concept that E. granulosus reduces cancer growth. This can be by a direct effect or indirectly by the development of immunity against common antigens associated with cancer and echinococcosis.

\section{Echinococcus AND ADAPTIVE IMMUNITY}

In an early study, antigenic similarity was reported between pulmonary carcinoma and hydatid cyst fluid (11). However, common antigens present in E. granulosus and some tumor types were thought to modulate host immune responses inducing anticancer activity (12). Cancers and parasites share similar properties in that both express mucin-type O-glycans, which are not usually found on healthy cell surfaces $(13,14)$. O-glycans present in cancer cells play key roles in metastasis, cell adhesion, and invasion (15). Cancer-associated O-glycosylated Tn ( $\alpha$ - $N$-acetylgalactosamine-O-serine/threonine) antigens have been detected in both larval and adult E. granulosus worm extracts with most activity recorded in the adult excretory/secretory (ES) products (16). More recently, antigens, which may 
be mucin type O-glycans, have been identified in hydatid cyst fluid, in laminated and germinal layers, and in the ES products of hydatid cyst protoscoleces (12). Immunological cross-reactivity of hydatid cyst fluid antigens with sera from cancer patients has been reported to be at an unusually higher level than in sera from healthy individuals (17). Therefore, antibody-mediated immune responses induced by these Tn antigens in echinococcosis patients are considered to induce immunity against cancer growth (12). Mucin-like peptides from E. granulosus (Egmuc) have been shown to induce an increase in activated natural killer (NK) cells in the spleens of immunized mice in a process mediated by soluble dendritic cell-derived factors (18). In vivo primed-splenocytes with Egmuc peptides induce pancreatic tumor cell cytotoxicity in vitro. However Egmuc-specific antibodies hardly recognized tumor derived antigens; therefore, the anticancer effects of Egmuc are probably due to its stimulating NK cell activation and inducing a Th1-like response (18). In contrast to control sera from people with no history of echinococcosis, sera from patients with hydatid disease have a cytotoxic effect on human lung small cell carcinoma cells (19) providing additional evidence of antibodymediated immunity against cancer. Furthermore, $40 \%$ of mice vaccinated with hydatid cyst fluid were shown to induce tumor regression in a colon cancer model and also induced an adaptive immune response against tumor re-challenge (20).

An experimental breast cancer model study, where rats had induced mammary carcinogenesis, showed that animals 20 days post echinococcosis infection showed reduced tumor growth compared with uninfected rats (21). Overall, these studies suggest that some E. granulosus antigens can induce memory cell formation to attack similar cancer-associated antigens. A proteomics study identified two proteins, mortalin (GRP75) and creatine kinase M-type, as being present both in E. granulosus and in colon cancer (20), and it has been reported that intratumoral and intraperitoneal injections of antiGRP75 antibodies suppressed tumor growth (22). In contrast, a monoclonal antibody developed against a $40 \mathrm{kDa}$ band in E. granulosus hydatid cyst fluid, which bound serum from breast cancer patients, had no significant effect on the growth of breast cancer cells in vitro (23).

Experiments in vitro have shown that neutrophils are actively involved in the killing of E. granulosus oncospheres (eggs; the infective stages to humans and ungulates) indicating an antibodydependent, cell mediated response (24). However, in progressed malignancy, a substantial antitumor immune response is needed to eliminate cancer cells (25). A hallmark of immunotherapy is long-term memory of the adaptive immune response (26). This may be an explanation why echinococcosis patients in endemic areas develop resistance to cancer (7). In direct contrast, injection of 4T1 mouse breast cancer cells into mice with experimental secondary echinococcosis resulted in an increased level of cancer metastasis in the liver, which was associated with a reduced Th1 immune response (9).

Overall, many studies are in favor of using E. granulosus antigens in cancer therapy. However more research is needed to identify the specific molecules of this parasite which reduce the cancer risk and/or can act as potential future treatments.

\section{CANCER KILLING IMMUNE RESPONSES AGAINST ECHINOCOCCOSIS}

Cancer cells are able to evade host immunity through various mechanisms, eventually establishing a relationship that mimics a chronic infection (27). Following their initial recognition, Th-1 polarized lymphocytes activate cytotoxic $\mathrm{T}$ cells and macrophages to destroy cancer cells (28). There is evidence showing that a Th-1 polarized response is protective against several cancers whereas patients with a polarized Th-2 response have poor prognosis in breast, lung, colorectal, and pancreatic cancers (29). In the early stages of echinococcosis, a Th1 immune response dominates, but during cyst establishment and growth, there is a switch to a Th-2 response, which is beneficial to the parasite for survival (30). When the hydatid cyst is either dying or dead, the Th2 response wanes rapidly allowing a Th1 response to take over (30).

As the recognition of specific tumor-associated antigens is the crucial step initiating an antitumor immune response (31), exposure to cancer-like antigens expressed by the Echinococcus parasites can stimulate such an anti-cancer response.

\section{DIRECT CANCER CELL KILLING BY Echinococcus}

Apart from generating antibody-mediated immunity, there is some evidence suggesting that Echinococcus parasites can directly kill cancer cells. Hydatid cyst protoscoleces have been shown to inhibit the proliferation of baby hamster kidney fibroblasts and induce the death of fibrosarcoma cells in vitro (5), although the specific molecules involved are not known. In an in vivo study in C57BL/6 mice, treatment with hydatid cyst fluid concurrently with the injection of melanoma cells resulted in a reduction in tumor growth (32). However, it was not clear in this study whether the control mouse group also received alum as an adjuvant control either injected intraperitoneally or in to the tumor margin (32). A potential concern is that being an aluminium-based adjuvant, alum can selectively stimulate a Th2 immune response in mice (33) which might play a role in anti-cancer effects (34).

EgKI-1 is a recently identified potent Kunitz type protease inhibitor highly expressed in oncospheres of E. granulosus (35). EgKI-1 treatment inhibits the growth and migration of a variety of cancer cells in vitro by negatively affecting cell cycle progression causing apoptosis. ${ }^{1}$ Furthermore, EgKI-1 treatment significantly reduced tumor growth in a triple negative breast cancer model (see text footnote 1).

\section{NEUTROPHILS AND ECHINOCOCCOSIS}

There is marked activity of cell-mediated immunity during the acute phase of echinococcosis including the infiltration of

${ }^{1}$ Ranasinghe SL, Boyle GM, Fischer K, Potriquet J, Mulvenna JP, McManus DP. EgKI-1, a promising anti-cancer therapeutic from the canine tapeworm Echinococcus granulosus. Sci Rep. (Under Review). 
inflammatory cells, which mainly comprise neutrophils and macrophages (30). Proteases such as neutrophil elastase (NE), secreted by activated neutrophils, can digest foreign parasite bodies and induce neutrophil chemotaxis. As a potent $\mathrm{NE}$ inhibitor of the secretory type, EgKI-1 from the oncospheres of E. granulosus might protect this stage from the host immune system (35).

Furthermore, in the chronic stage of echinococcosis, if the hydatid cyst ruptures, neutrophils are attracted to kill the contained protoscoleces, and this might be a reason for the elevated expression of Antigen B (AgB) in hydatid cyst fluid (36). Being another potent protease inhibitor, AgB can significantly reduce neutrophil recruitment, thus delaying the potential killing of protoscoleces by neutrophils until the larvae can grow into larger cysts resulting in secondary echinococcosis. Inhibiting NE secretion and neutrophil chemotaxis is, therefore, important for E. granulosus survival during both the acute and chronic disease phases (Figure 1).

\section{NEUTROPHILS AND CANCER}

While neutrophils play a major role in host defense, these cells have both pro- and antitumor effects in cancer patients (37). Many subjects with advanced cancers show high numbers of neutrophils in their blood (38) even though the precise mechanisms involved are unknown. Recent evidence has indicated that neutrophils in the tumor microenvironment actively contribute to tumor growth initiation, progression, metastasis, and angiogenesis (39-41) (Figure 2). Consequently, potent NE inhibitors have been tested as anticancer therapeutics (42). As indicated above, as a potent NE inhibitor, the EgKI-1 protein exhibits anticancer effects both in vitro and in vivo (see text footnote 1).

\section{Echinococcus ANTIGENS IN CANCER THERAPY}

As referred to earlier, there is evidence suggesting that some Echinococcus antigens have the capacity to induce antibodymediated immunity, which can induce non-specific immunity against certain cancer types, whereas the EgKI-1 protein secreted by $E$. granulosus oncospheres is able to kill cancer cells directly. As AgB is also a potent NE inhibitor, it would be profitable to investigate whether it can also kill cancer cells. However, apart from NE inhibition, other mechanisms and/or molecules, which interact with EgKI-1 causing the observed anticancer effects in vivo, are likely involved and still need to be investigated. Furthermore, bioavailability and serum clearance can determine how released molecules from a parasite such as Echinococcus act in vivo although it is known that the "excretory/secretory" products from helminth worms can circulate in the body through the lymph or blood (28).

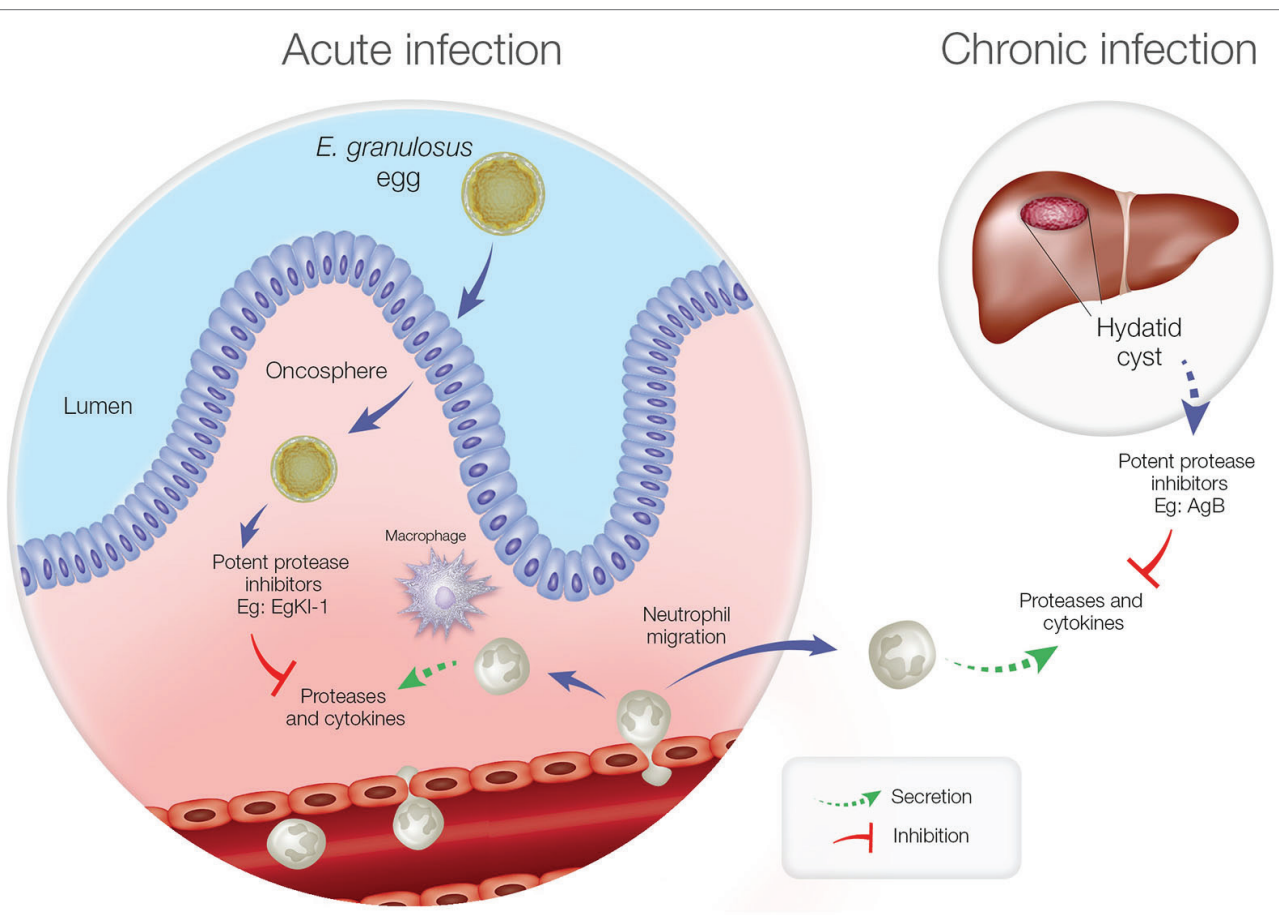

FIGURE 1 | Neutrophil function during acute and chronic echinococcosis. In the acute stage, neutrophils and macrophages migrate to the intestinal mucosa to attack invading oncospheres. In the chronic stage, if hydatid cyst fluid leakage occurs from a ruptured cyst, neutrophils are attracted but antigen B inhibits neutrophil chemotaxis and neutrophil elastase to protect protoscoleces so they can develop into new cysts. 

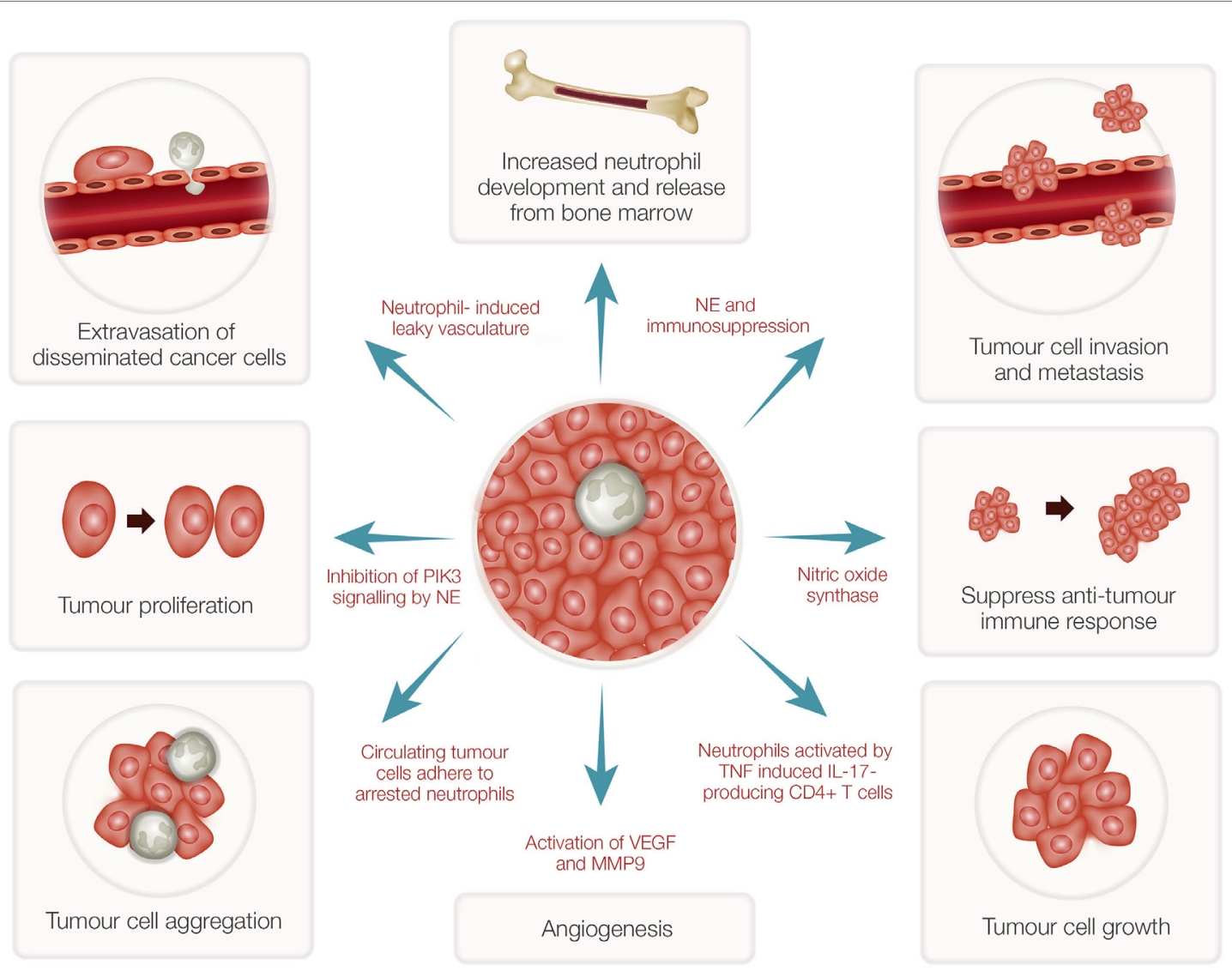

FIGURE 2 | Tumor-associated neutrophils (TANs) and cancer growth. TANs can stimulate bone marrow to develop and release more neutrophils. Reactive oxygen species and proteases secreted from activated neutrophils can induce epithelial damage and subsequent tumor promoting inflammation. Nitric oxide synthase expressed by neutrophils suppresses $\mathrm{CD} 8^{+} \mathrm{T}$-cell-mediated antitumor immune responses thus stimulating tumor progression. Increased neutrophils can induce leaky vasculature facilitating extravasation of disseminated cancer cells and aggregation leading to tumor metastasis. Neutrophil elastase (NE), secreted by activated neutrophils, can inhibit phosphoinositide 3-kinase (PIK3) signaling, and suppress immune reactions leading to tumor growth and proliferation. Further, neutrophils induce angiogenesis by activation of vascular endothelial growth factor (VEGF) mediated by matrix metalloproteinase 9 (MMP9).

\section{CONCLUSION}

Scrutiny of the available literature suggests that certain Echinococcus antigens can generate adaptive immunity against cancer. Moreover EgKI-1, which is secreted by E. granulosus, shows direct anticancer effects. Therefore, this canine tapeworm may actually provide some hope as a potential cure against some forms of cancer. Additional studies are now required to progress this research further and to identify additional specific proteins secreted by this tapeworm for application in future anticancer therapy.

\section{REFERENCES}

1. Oikonomopoulou K, Brinc D, Kyriacou K, Diamandis EP. Infection and cancer: revaluation of the hygiene hypothesis. Clin Cancer Res (2013) 19(11):2834-41. doi:10.1158/1078-0432.CCR-12-3661

2. Atayde VD, Jasiulionis MG, Cortez M, Yoshida N. A recombinant protein based on Trypanosoma cruzi surface molecule gp 82 induces apoptotic cell death in melanoma cells. Melanoma Res (2008) 18(3):172-83. doi:10.1097/ CMR.0b013e3282feeaab

\section{AUTHOR CONTRIBUTIONS}

SR drafted the manuscript and DM critically evaluated and edited the manuscript.

\section{FUNDING}

This study was funded by a program grant from the National Health and Medical Research Council of Australia (NHMRC) to DPM. DPM is a senior principal research fellow of the NHMRC.

3. Shirzad H, Khorami S, Soozangar N, Yousefi M, Darani HY. Toxoplasma gondii but not Leishmania major or Trichomonas vaginalis decreases cell proliferation and increases cell death on fibrosarcoma cancer cells in culture medium. WJV (2012) 2(2):105. doi:10.4236/wjv.2012.22014

4. Pidherney MS, Alizadeh H, Stewart GL, McCulley JP, Niederkorn JY. In vitro and in vivo tumoricidal properties of a pathogenic/free-living amoeba. Cancer Lett (1993) 72(1-2):91-8. doi:10.1016/0304-3835(93)90016-3

5. Yousofi Darani H, Soozangar N, Khorami S, Taji F, Yousofi M, Shirzad H. Hydatid cyst protoscolices induce cell death in WEHI-164 fibrosarcoma 
cells and inhibit the proliferation of baby hamster kidney fibroblasts in vitro. J Parasitol Res (2012) 2012:304183. doi:10.1155/2012/304183

6. Plumelle Y, Gonin C, Edouard A, Bucher BJ, Thomas L, Brebion A, et al. Effect of Strongyloides stercoralis infection and eosinophilia on age at onset and prognosis of adult T-cell leukemia. Am J Clin Pathol (1997) 107(1):81-7. doi:10.1093/ajcp/107.1.81

7. Akgul H, Tez M, Unal AE, Keskek M, Sayek I, Ozcelik T. Echinococcus against cancer: why not? Cancer (2003) 98(9):1999-2000. doi:10.1002/cncr. 11752

8. Tez S, Tez M. Echinococcus and cancer: unsolved mystery. Parasite Immunol (2015) 37(8):426. doi:10.1111/pim.12201

9. Turhan N, Esendagli G, Ozkayar O, Tunali G, Sokmensuer C, Abbasoglu O. Co-existence of Echinococcus granulosus infection and cancer metastasis in the liver correlates with reduced Th1 immune responses. Parasite Immunol (2015) 37(1):16-22. doi:10.1111/pim.12152

10. Oikonomopoulou K, Yu H, Wang Z, Vasiliou SK, Brinc D, Christofi G, et al. Association between Echinococcus granulosus infection and cancer risk-a pilot study in Cyprus. Clin Chem Lab Med (2016) 54(12):1955-61. doi:10.1515/cclm-2016-0125

11. Yong W, Heath D, Savage T. Possible antigenic similarity between pulmonary carcinoma and cysts of Echinococcus granulosus. Br Med J (1979) 1(6176):1463. doi:10.1136/bmj.1.6176.1463-a

12. Daneshpour S, Bahadoran M, Hejazi SH, Eskandarian AA, Mahmoudzadeh M, Darani HY. Common antigens between hydatid cyst and cancers. Adv Biomed Res (2016) 5:9. doi:10.4103/2277-9175.175242

13. Osinaga E. Expression of cancer-associated simple mucin-type O-glycosylated antigens in parasites. IUBMB Life (2007) 59(4-5):269-73. doi:10.1080/ 15216540601188553

14. Rumyantsev SN. Evolutionary adaptations of human cancer for parasitic life. Open J Immunol (2013) 3(02):54. doi:10.4236/oji.2013.32009

15. Pinho SS, Reis CA. Glycosylation in cancer: mechanisms and clinical implications. Nat Rev Cancer (2015) 15(9):540-55. doi:10.1038/nrc3982

16. Errico DA, Medeiros A, Miguez M, Casaravilla C, Malgor R, Carmona C, et al. O-glycosylation in Echinococcus granulosus: identification and characterization of the carcinoma-associated Tn antigen. Exp Parasitol (2001) 98(2):100-9. doi:10.1006/expr.2001.4620

17. Pfister M, Gottstein B, Cerny T, Cerny A. Immunodiagnosis of echinococcosis in cancer patients. Clin Microbiol Infect (1999) 5(11):693-7. doi:10.111 1/j.1469-0691.1999.tb00515.x

18. Noya V, Bay S, Festari MF, García EP, Rodriguez E, Chiale C, et al. Mucin-like peptides from Echinococcus granulosus induce antitumor activity. Int $J$ Oncol (2013) 43(3):775-84. doi:10.3892/ijo.2013.2000

19. Karadayi S, Arslan S, Sumer Z, Turan M, Sumer H, Karadayi K. Does hydatid disease have protective effects against lung cancer? Mol Biol Rep (2013) 40(8):4701-4. doi:10.1007/s11033-013-2565-8

20. Berriel E, Russo S, Monin L, Festari MF, Berois N, Fernandez G, et al. Antitumor activity of human hydatid cyst fluid in a murine model of colon cancer. ScientificWorldJournal (2013) 2013:230176. doi:10.1155/2013/230176

21. Altun A, Saraydin SU, Soylu S, Inan DS, Yasti C, Ozdenkaya Y, et al. Chemopreventive effects of hydatiddisease on experimentalbreastcancer.Asian Pac J Cancer Prev (2015) 16(4):1391-5. doi:10.7314/APJCP.2015.16.4.1391

22. Gundogdu SB, Saylam B, Tez M. Cyst hydatid and cancer: the myth continues. Clin Chem Lab Med (2017) 55(7):e150-1. doi:10.1515/cclm-2016-0626

23. Sharafi SM, Shirzad H, Khanahmad H, Ataei B, Darani HY. Monoclonal antibodies production against a $40 \mathrm{KDa}$ band of hydatid cyst fluid. Recent Pat Biotechnol (2018) 12(1):57-64. doi:10.2174/1872208311666170317151346

24. Rogan M, Craig P, Zehyle E, Masinde G, Wen H, Zhou P. In vitro killing of taeniid oncospheres, mediated by human sera from hydatid endemic areas. Acta Trop (1992) 51(3-4):291-6. doi:10.1016/0001-706X(92)90047-2

25. Dunn GP, OldLJ,SchreiberRD. The threeEsof cancerimmunoediting. AnnuRev Immunol (2004) 22:329-60. doi:10.1146/annurev.immunol.22.012703.104803
26. Sharma P, Hu-Lieskovan S, Wargo JA, Ribas A. Primary, adaptive, and acquired resistance to cancer immunotherapy. Cell (2017) 168(4):707-23. doi:10.1016/j.cell.2017.01.017

27. Goldszmid RS, Dzutsev A, Trinchieri G. Host immune response to infection and cancer: unexpected commonalities. Cell Host Microbe (2014) 15(3): 295-305. doi:10.1016/j.chom.2014.02.003

28. Hewitson JP, Grainger JR, Maizels RM. Helminth immunoregulation: the role of parasite secreted proteins in modulating host immunity. Mol Biochem Parasitol (2009) 167(1):1-11. doi:10.1016/j.molbiopara.2009.04.008

29. Lippitz BE. Cytokine patterns in patients with cancer: a systematic review. Lancet Oncol (2013) 14(6):e218-28. doi:10.1016/S1470-2045(12)70582-X

30. Zhang W, Ross AG, McManus DP. Mechanisms of immunity in hydatid disease: implications for vaccine development. J Immunol (2008) 181(10):6679-85. doi:10.4049/jimmunol.181.10.6679

31. Jacqueline C, Tasiemski A, Sorci G, Ujvari B, Maachi F, Misse D, et al. Infections and cancer: the "fifty shades of immunity" hypothesis. BMC Cancer (2017) 17(1):257. doi:10.1186/s12885-017-3234-4

32. Darani HY, Sharafi SM, Mokarian F, Yousefi M, Sharafi SA, Jafari R. Therapeutic effect of hydatid cyst liquid on melanoma tumor growth in mouse model. $\mathrm{Br}$ J Med Med Res (2016) 18(2):1-7. doi:10.9734/BJMMR/2016/27220

33. Hogenesch H. Mechanism of immunopotentiation and safety of aluminum adjuvants. Front Immunol (2012) 3:406. doi:10.3389/fimmu.2012.00406

34. Ellyard JI, Simson L, Parish CR. Th2-mediated anti-tumour immunity: friend or foe? Tissue Antigens (2007) 70(1):1-11. doi:10.1111/j.1399-0039.2007. 00869.x

35. Ranasinghe SL, Fischer K, Zhang W, Gobert GN, McManus DP. Cloning and characterization of two potent Kunitz type protease inhibitors from Echinococcus granulosus. PLoS Negl Trop Dis (2015) 9(12):e0004268. doi:10.1371/journal.pntd.0004268

36. Shepherd JC, Aitken A, McManus DP. A protein secreted in vivo by Echinococcus granulosus inhibits elastase activity and neutrophil chemotaxis. Mol Biochem Parasitol (1991) 44(1):81-90. doi:10.1016/0166-6851(91)90223-S

37. Treffers LW, Hiemstra IH, Kuijpers TW, van den Berg TK, Matlung HL. Neutrophils in cancer. Immunol Rev (2016) 273(1):312-28. doi:10.1111/ imr.12444

38. Schmidt H, Bastholt L, Geertsen P, Christensen IJ, Larsen S, Gehl J, et al. Elevated neutrophil and monocyte counts in peripheral blood are associated with poor survival in patients with metastatic melanoma: a prognostic model. Br J Cancer (2005) 93(3):273-8. doi:10.1038/sj.bjc.6602702

39. Coffelt SB, Wellenstein MD, de Visser KE. Neutrophils in cancer: neutral no more. Nat Rev Cancer (2016) 16(7):431-46. doi:10.1038/nrc.2016.52

40. Tuting T, de Visser KE. CANCER. How neutrophils promote metastasis. Science (2016) 352(6282):145-6. doi:10.1126/science.aaf7300

41. Zhang X, Zhang W, Yuan X, Fu M, Qian H, Xu W. Neutrophils in cancer development and progression: roles, mechanisms, and implications (review). Int J Oncol (2016) 49(3):857-67. doi:10.3892/ijo.2016.3616

42. Inada $M$, Yamashita J, Ogawa $M$. Neutrophil elastase inhibitor (ONO5046-Na) inhibits the growth of human lung cancer cell lines transplanted into severe combined immunodeficiency (scid) mice. Res Commun Mol Pathol Pharmacol (1997) 97(2):229-32.

Conflict of Interest Statement: The authors declare that the research was conducted in the absence of any commercial or financial relationships that could be construed as a potential conflict of interest.

Copyright $\odot 2018$ Ranasinghe and McManus. This is an open-access article distributed under the terms of the Creative Commons Attribution License (CC BY). The use, distribution or reproduction in other forums is permitted, provided the original author(s) and the copyright owner are credited and that the original publication in this journal is cited, in accordance with accepted academic practice. No use, distribution or reproduction is permitted which does not comply with these terms. 